Bull. Korean Math. Soc. 50 (2013), No. 5, pp. 1659-1671

http://dx.doi.org/10.4134/BKMS.2013.50.5.1659

\title{
WAITING TIME DISTRIBUTION IN THE M/M/M RETRIAL QUEUE
}

\author{
JeOngSim Kim AND JeRIM Kim
}

\begin{abstract}
In this paper, we are concerned with the analysis of the waiting time distribution in the $\mathrm{M} / \mathrm{M} / \mathrm{m}$ retrial queue. We give expressions for the Laplace-Stieltjes transform (LST) of the waiting time distribution and then provide a numerical algorithm for calculating the LST of the waiting time distribution. Numerical inversion of the LSTs is used to calculate the waiting time distribution. Numerical results are presented to illustrate our results.
\end{abstract}

\section{Introduction}

Retrial queues are queueing systems in which arriving customers who find all servers occupied may retry for service again after a random amount of time. Retrial queues have been widely used to model many problems in telephone systems, call centers, telecommunication networks, computer networks and computer systems, and in daily life. Detailed overviews for retrial queues can be found in the bibliographies $[2,3,4]$, the surveys $[9,17,20]$, and the books $[6,10]$.

In this paper we consider an $\mathrm{M} / \mathrm{M} / \mathrm{m}$ retrial queue. Multi-server retrial queues are characterized by the following features: If there is a free server when a customer arrives from outside the system, this customer begins to be served immediately and leaves the system after the service is completed. On the other hand, any customer who finds all the servers busy upon arrival joins a retrial group, called an orbit, and then attempts service after a random amount of time. If there is a free server when a customer from the orbit attempts service, this customer receives service immediately and leaves the system after the service completion. Otherwise the customer comes back to the orbit immediately and repeats the retrial process.

Received October 22, 2012; Revised February 25, 2013.

2010 Mathematics Subject Classification. Primary 60K25.

Key words and phrases. M/M/m retrial queue, waiting time, Laplace-Stieltjes transform, first passage time.

The first author's research was supported by Basic Science Research Program through the National Research Foundation of Korea (NRF) funded by the Ministry of Education, Science and Technology (NRF-2011-0011887). 
The $\mathrm{M} / \mathrm{M} / \mathrm{m}$ retrial queue has been studied by several authors. Most of the existing literature for the $\mathrm{M} / \mathrm{M} / \mathrm{m}$ retrial queue concentrates on the steadystate probability of the number of customers in the orbit. Due to the complexity of the analysis, no explicit closed-form formulas are obtained for the steady-state probability of a retrial queue with more than two servers. Keilson et al. [14] established a recursive algorithm for the computation of steady-state probabilities in the $\mathrm{M} / \mathrm{M} / 2$ retrial queue. Hanschke [13] showed that the generating functions of the steady-state probabilities can be expressed in terms of generalized hypergeometric functions in the $\mathrm{M} / \mathrm{M} / 2$ retrial queue. For the $\mathrm{M} / \mathrm{M} / \mathrm{m}$ retrial queue, several approximations and numerical methods have been proposed (see, for example, $[11,18,19]$ ).

In this paper, we focus on the analysis of the waiting time distribution. The distribution of the waiting time for an arbitrary customer in retrial queues is much more difficult to analyze than that of the number of customers in the orbit. The analysis of the waiting time distribution is intricate because customers in the orbit operate under random order service discipline. For the M/M/1 retrial queue, Falin [8] gave in the form of an integro-differential equation for the Laplace-Stieltjes transform (LST) of the waiting time distribution. The equation was very difficult to solve. The author gave only the first two moments of the waiting time distribution. Hanschke [12] developed a recursive procedure for the computation of variance of the waiting time distribution in the $\mathrm{M} / \mathrm{M} / 1$ retrial queue. Choo and Conolly [7] presented a closed-form formula for the LST of the waiting time distribution in the $\mathrm{M} / \mathrm{M} / 1$ retrial queue, though there were some errors in their analysis [16].

To the best of our knowledge, there are no known analytic results for the waiting time in the $\mathrm{M} / \mathrm{M} / \mathrm{m}$ retial queue with $m \geq 2$. Since the study of $\mathrm{M} / \mathrm{M} / \mathrm{m}$ queue with infinite retrial group seems intractable, Artalejo and Gómez-Corral [5] developed algorithmic procedure for the computation of the waiting time distribution in the $\mathrm{M} / \mathrm{M} / \mathrm{m}$ queue with finite retrial group.

The purpose of this paper is to obtain the analytic results that are practically useful for the numerical calculation of the waiting time distribution in the $\mathrm{M} / \mathrm{M} / \mathrm{m}$ retrial queue. In Section 3, we give expressions for the conditional transforms of the first passage time distributions which play a crucial role in obtaining the LST of the waiting time distribution. In Section 4, we give expressions for the LSTs of the conditional waiting time and the unconditional waiting time. In Section 5, we provide a numerical algorithm for calculating the LST of the waiting time distribution and use the numerical inversion of the LSTs to calculate the waiting time distribution. Numerical results are presented in Section 6.

\section{The model}

We consider the $\mathrm{M} / \mathrm{M} / \mathrm{m}$ retrial queue where customers arrive from outside the system according to a Poisson process with rate $\lambda$. The service facility 
consists of $m$ identical servers, and service times are exponentially distributed with mean $\mu^{-1}$. The retrial time, i.e., the length of the time interval between two consecutive attempts made by a customer in the orbit, is exponentially distributed with mean $\nu^{-1}$. The arrival process, the service times, and the retrial times are assumed to be mutually independent. The offered load $\rho$ is defined as $\rho=\frac{\lambda}{m \mu}$. We assume that $\rho<1$ for stability of the system.

Let $N(t)$ denote the number of customers in the orbit at time $t$ and $S(t)$ the number of busy servers at time $t$. Then $\{(N(t), S(t)): t \geq 0\}$ is a continuous time Markov process with state space $\{(n, i): n \geq 0,0 \leq i \leq m\}$. We assume that every sample path of the Markov process is right continuous. The infinitesimal generator $Q$ of the process $\{(N(t), S(t)): t \geq 0\}$ is

$$
Q=\left[\begin{array}{cccccc}
B-C & E & & & & \\
\\
A & B-C-D & E & & & \\
& 2 A & B-C-2 D & E & & \\
& & 3 A & B-C-3 D & E & \\
& & & \ddots & \ddots & \ddots
\end{array}\right],
$$

where $A, B, C, D$ and $E$ are $(m+1) \times(m+1)$ matrices whose $(i, j)$-components $A_{i j}, B_{i j}, C_{i j}, D_{i j}$, and $E_{i j}$ are given by

$$
\begin{aligned}
& A_{i j}= \begin{cases}\nu & \text { if } 1 \leq j=i+1 \leq m, \\
0 & \text { otherwise, }\end{cases} \\
& B_{i j}=\left\{\begin{array}{cc}
\lambda & \text { if } 1 \leq j=i+1 \leq m, \\
i \mu & \text { if } 1 \leq i=j+1 \leq m, \\
0 & \text { otherwise, }
\end{array}\right. \\
& C_{i j}=\left\{\begin{array}{cc}
\lambda+i \mu & \text { if } 0 \leq i=j \leq m, \\
0 & \text { otherwise, }
\end{array}\right. \\
& D_{i j}= \begin{cases}\nu & \text { if } 0 \leq i=j \leq m-1, \\
0 & \text { otherwise }\end{cases} \\
& E_{i j}= \begin{cases}\lambda & \text { if } i=j=m, \\
0 & \text { otherwise. }\end{cases}
\end{aligned}
$$

Let

$$
\pi_{n i}=\lim _{t \rightarrow \infty} \mathbb{P}((N(t), S(t))=(n, i))
$$

be the joint distribution of the number of customers in the orbit and the number of busy servers in the steady state and let $\boldsymbol{\pi}_{n}=\left(\pi_{n 0}, \ldots, \pi_{n m}\right)$.

The main interest of this paper is to obtain analytic results that are practically useful for the numerical calculation of the waiting time distribution. When there is at least one customer initially present in the orbit, i.e., when $N(0) \geq 1$, we choose an arbitrary customer and call it a 'tagged customer'. Let $\sigma$ be the service initiation epoch of the tagged customer. Also let $\mathcal{W}$ denote a random variable representing the waiting time of the tagged customer. By the 
Poisson arrivals see time averages (PASTA) property, the waiting time $\mathcal{W}$ of the tagged customer has the distribution

(1) $\mathbb{P}(\mathcal{W} \leq x)=1-\sum_{n=0}^{\infty} \pi_{n m}+\sum_{n=0}^{\infty} \pi_{n m} \mathbb{P}(\sigma \leq x \mid N(0)=n+1, S(0)=m)$.

\section{First passage time distributions}

Define

$$
\tau_{n}=\inf \{t>0: N(t)=n\}, \quad n=1,2,3, \ldots,
$$

to be the first time that there are $n$ customers present in the orbit. Now we introduce the conditional transforms related to $\tau_{n}$ and $\sigma$. For $n \geq 1$, let

$$
\begin{aligned}
\left(G_{n}(s)\right)_{i j} & =\mathbb{E}\left[e^{-s \tau_{n-1}} \mathbb{1}_{\left\{\tau_{n-1}<\sigma, S\left(\tau_{n-1}\right)=j\right\}} \mid N(0)=n, S(0)=i\right], 0 \leq i, j \leq m, \\
\left(h_{n}(s)\right)_{i} & =\mathbb{E}\left[e^{-s \sigma} \mathbb{1}_{\left\{\tau_{n-1} \geq \sigma\right\}} \mid N(0)=n, S(0)=i\right], 0 \leq i \leq m .
\end{aligned}
$$

Let $G_{n}(s)$ be the $(m+1) \times(m+1)$ matrix whose components are $\left(G_{n}(s)\right)_{i j}$, $0 \leq i, j \leq m$, and let $\boldsymbol{h}_{n}(s)$ be the $(m+1)$-dimensional column vector whose components are $\left(h_{n}(s)\right)_{i}, 0 \leq i \leq m$. Then $G_{n}(s)$ and $\boldsymbol{h}_{n}(s)$ satisfy the following theorem.

Theorem 1. For complex numbers $s$ with $\operatorname{Re}(s) \geq 0$,

$$
\begin{aligned}
G_{n}(s) & =(n-1)\left[C+n D-B-E G_{n+1}(s)+s I\right]^{-1} A, \\
\boldsymbol{h}_{n}(s) & =\left[C+n D-B-E G_{n+1}(s)+s I\right]^{-1}\left(A \mathbf{1}+E \boldsymbol{h}_{n+1}(s)\right),
\end{aligned}
$$

where $\mathbf{1}$ is an $(m+1)$-dimensional column vector with all its components equal to one.

Proof. We define the first transition epoch out of the initial state for the process $\{(N(t), S(t)): t \geq 0\}$, i.e., let

$$
t_{0}=\inf \{t>0:(N(t), S(t)) \neq(N(0), S(0))\} .
$$

Let $J$ be defined as

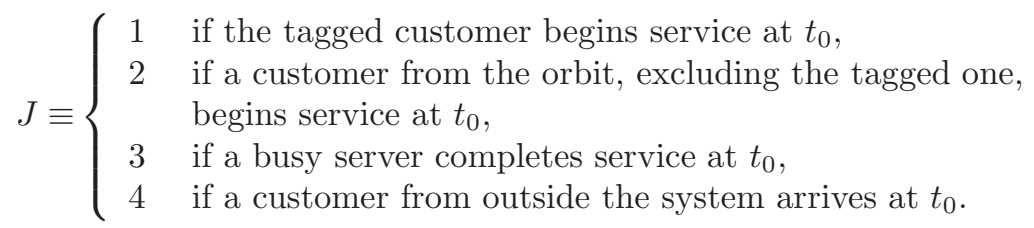

Then

(4)

$$
\begin{aligned}
= & \sum_{l=1}^{4} \mathbb{P}(J=l \mid N(0)=n, S(0)=i) \\
& \times \mathbb{E}\left[e^{-s \tau_{n-1}} \mathbb{1}_{\left\{\tau_{n-1}<\sigma, S\left(\tau_{n-1}\right)=j\right\}} \mid N(0)=n, S(0)=i, J=l\right]
\end{aligned}
$$




$$
\begin{aligned}
= & \sum_{l=1}^{4} \mathbb{P}(J=l \mid N(0)=n, S(0)=i) \mathbb{E}\left[e^{-s t_{0}} \mid N(0)=n, S(0)=i, J=l\right] \\
& \times \mathbb{E}\left[e^{-s\left(\tau_{n-1}-t_{0}\right)} \mathbb{1}_{\left\{\tau_{n-1}-t_{0}<\sigma-t_{0}, S\left(\tau_{n-1}\right)=j\right\}} \mid N(0)=n, S(0)=i, J=l\right],
\end{aligned}
$$

where the last equality follows from the fact that $t_{0}$ and $\left(\tau_{n-1}-t_{0}, \sigma-t_{0}\right.$, $S\left(\tau_{n-1}\right)$ ) are independent, given $\{N(0)=n, S(0)=i, J=l\}, 1 \leq l \leq 4$. Given $\{N(0)=n, S(0)=i\}, 0 \leq i \leq m, t_{0}$ has the exponential distribution with mean $\left((C+n D)_{i i}\right)^{-1}$ and is independent of $J$. Hence, for every complex number $s$ with $\operatorname{Re}(s) \geq 0,1 \leq l \leq 4$ and $0 \leq i \leq m$,

$$
\mathbb{E}\left[e^{-s t_{0}} \mid N(0)=n, S(0)=i, J=l\right]=\frac{(C+n D)_{i i}}{(C+n D+s I)_{i i}} .
$$

Furthermore, given $\{N(0)=n, S(0)=i\}, 0 \leq i \leq m, J$ has the following distributions:

$$
\left\{\begin{array}{l}
\mathbb{P}(J=1 \mid N(0)=n, S(0)=i)=\frac{\nu\left(1-\delta_{i m}\right)}{(C+n D)_{i i}}, \\
\mathbb{P}(J=2 \mid N(0)=n, S(0)=i)=\frac{(n-1) \nu\left(1-\delta_{i m}\right)}{(C+n D)_{i i}}, \\
\mathbb{P}(J=3 \mid N(0)=n, S(0)=i)=\frac{i \mu}{(C+n D)_{i i}}, \\
\mathbb{P}(J=4 \mid N(0)=n, S(0)=i)=\frac{\lambda}{(C+n D)_{i i}},
\end{array}\right.
$$

with $\delta_{i m}$ being the Kronecker delta. Substituting (5) and (6) into (4) yields (7)

$$
\begin{aligned}
& \left(G_{n}(s)\right)_{i j} \\
= & \frac{\nu\left(1-\delta_{i m}\right)}{(C+n D+s I)_{i i}} \mathbb{E}\left[e^{-s\left(\tau_{n-1}-t_{0}\right)} \mathbb{1}_{\left\{\tau_{n-1}-t_{0}<\sigma-t_{0}, S\left(\tau_{n-1}\right)=j\right\}} \mid N(0)=n, S(0)=i, J=1\right] \\
& +\frac{(n-1) \nu\left(1-\delta_{i m}\right)}{(C+n D+s I)_{i i}} \mathbb{E}\left[e^{-s\left(\tau_{n-1}-t_{0}\right)} \mathbb{1}_{\left\{\tau_{n-1}-t_{0}<\sigma-t_{0}, S\left(\tau_{n-1}\right)=j\right\}} \mid N(0)=n, S(0)=i, J=2\right] \\
& +\frac{i \mu}{(C+n D+s I)_{i i}} \mathbb{E}\left[e^{-s\left(\tau_{n-1}-t_{0}\right)} \mathbb{1}_{\left\{\tau_{n-1}-t_{0}<\sigma-t_{0}, S\left(\tau_{n-1}\right)=j\right\}} \mid N(0)=n, S(0)=i, J=3\right] \\
& +\frac{\lambda}{(C+n D+s I)_{i i}} \mathbb{E}\left[e^{-s\left(\tau_{n-1}-t_{0}\right)} \mathbb{1}_{\left\{\tau_{n-1}-t_{0}<\sigma-t_{0}, S\left(\tau_{n-1}\right)=j\right\}} \mid N(0)=n, S(0)=i, J=4\right] .
\end{aligned}
$$

Since $t_{0}=\tau_{n-1}=\sigma$ on $\{N(0)=n, S(0)=i, J=1\}, 0 \leq i \leq m-1$, the first conditional expectation on the right-hand side of (7) becomes

(8) $\mathbb{E}\left[e^{-s\left(\tau_{n-1}-t_{0}\right)} \mathbb{1}_{\left\{\tau_{n-1}-t_{0}<\sigma-t_{0}, S\left(\tau_{n-1}\right)=j\right\}} \mid N(0)=n, S(0)=i, J=1\right]=0$.

Since $t_{0}=\tau_{n-1}<\sigma$ and $S\left(t_{0}\right)=i+1$ on $\{N(0)=n, S(0)=i, J=2\}$, $0 \leq i \leq m-1$, the second conditional expectation on the right-hand side of $(7)$ becomes

$$
\text { (9) } \begin{aligned}
& \mathbb{E}\left[e^{-s\left(\tau_{n-1}-t_{0}\right)} \mathbb{1}_{\left\{\tau_{n-1}-t_{0}<\sigma-t_{0}, S\left(\tau_{n-1}\right)=j\right\}} \mid N(0)=n, S(0)=i, J=2\right] \\
= & \mathbb{E}\left[e^{-s\left(\tau_{n-1}-t_{0}\right)} \mathbb{1}_{\left\{S\left(t_{0}\right)=j\right\}} \mid N(0)=n, S(0)=i, S\left(t_{0}\right)=i+1\right] \\
= & \delta_{j, i+1} .
\end{aligned}
$$

For the third conditional expectation on the right-hand side of (7), we note that $N\left(t_{0}\right)=n$ and $S\left(t_{0}\right)=i-1$ on $\{N(0)=n, S(0)=i, J=3\}, 1 \leq$ $i \leq m$. Therefore, by the strong Markov property of the Markov process 
$\left\{\left(N(t), S(t), \mathbb{1}_{\{\sigma \geq t\}}\right): t \geq 0\right\}$, the third conditional expectation on the righthand side of (7) can be written as

(10)

$$
\begin{aligned}
& \mathbb{E}\left[e^{-s\left(\tau_{n-1}-t_{0}\right)} \mathbb{1}_{\left\{\tau_{n-1}-t_{0}<\sigma-t_{0}, S\left(\tau_{n-1}\right)=j\right\}} \mid N(0)=n, S(0)=i, J=3\right] \\
= & \mathbb{E}\left[e^{-s\left(\tau_{n-1}-t_{0}\right)} \mathbb{1}_{\left\{\tau_{n-1}-t_{0}<\sigma-t_{0}, S\left(\tau_{n-1}\right)=j\right\}} \mid N(0)=n, S(0)=i, N\left(t_{0}\right)=n,\right. \\
& \left.S\left(t_{0}\right)=i-1\right] \\
= & \mathbb{E}\left[e^{-s \tau_{n-1}} \mathbb{1}_{\left\{\tau_{n-1}<\sigma, S\left(\tau_{n-1}\right)=j\right\}} \mid N(0)=n, S(0)=i-1\right] \\
= & \left(G_{n}(s)\right)_{i-1, j} .
\end{aligned}
$$

Similarly, for $0 \leq i \leq m-1$,

$$
\begin{aligned}
& \mathbb{E}\left[e^{-s\left(\tau_{n-1}-t_{0}\right)} \mathbb{1}_{\left\{\tau_{n-1}-t_{0}<\sigma-t_{0}, S\left(\tau_{n-1}\right)=j\right\}} \mid N(0)=n, S(0)=i, J=4\right] \\
= & \left(G_{n}(s)\right)_{i+1, j},
\end{aligned}
$$

because $N\left(t_{0}\right)=n$ and $S\left(t_{0}\right)=i+1$ on $\{N(0)=n, S(0)=i, J=4\}, 0 \leq$ $i \leq m-1$. We also note that $N\left(t_{0}\right)=n+1$ and $S\left(t_{0}\right)=m$ on $\{N(0)=$ $n, S(0)=m, J=4\}$. Therefore, by the strong Markov property of Markov process $\left\{\left(N(t), S(t), \mathbb{1}_{\{\sigma \geq t\}}\right): t \geq 0\right\}$,

$$
\begin{aligned}
& \mathbb{E}\left[e^{-s\left(\tau_{n-1}-t_{0}\right)} \mathbb{1}_{\left\{\tau_{n-1}-t_{0}<\sigma-t_{0}, S\left(\tau_{n-1}\right)=j\right\}} \mid N(0)=n, S(0)=m, J=4\right] \\
= & \mathbb{E}\left[e^{-s \tau_{n-1}} \mathbb{1}_{\left\{\tau_{n-1}<\sigma, S\left(\tau_{n-1}\right)=j\right\}} \mid N(0)=n+1, S(0)=m\right] \\
= & \sum_{k=0}^{m} \mathbb{E}\left[e^{-s \tau_{n}} \mathbb{1}_{\left\{\tau_{n}<\sigma, S\left(\tau_{n}\right)=k\right\}} e^{-s\left(\tau_{n-1}-\tau_{n}\right)} \mathbb{1}_{\left\{\tau_{n-1}-\tau_{n}<\sigma-\tau_{n}, S\left(\tau_{n-1}\right)=j\right\}} \mid\right. \\
= & \sum_{k=0}^{m} \mathbb{E}\left[e^{-s \tau_{n}} \mathbb{1}_{\left\{\tau_{n}<\sigma, S\left(\tau_{n}\right)=k\right\}} \mid N(0)=n+1, S(0)=m\right] \\
& \times \mathbb{E}\left[e^{-s\left(\tau_{n-1}-\tau_{n}\right)_{1}} \mathbb{1}_{\left\{\tau_{n-1}-\tau_{n}<\sigma-\tau_{n}, S\left(\tau_{n-1}\right)=j\right\}} \mid N(0)=n+1, S(0)=m,\right. \\
\left.\quad N\left(\tau_{n}\right)=n, S\left(\tau_{n}\right)=k\right] & \sum_{k=0}^{m}\left(G_{n+1}(s)\right)_{m k}\left(G_{n}(s)\right)_{k j} .
\end{aligned}
$$

Therefore, the last conditional expectation on the right-hand side of (7) becomes

$$
\begin{aligned}
& \mathbb{E}\left[e^{-s\left(\tau_{n-1}-t_{0}\right)} \mathbb{1}_{\left\{\tau_{n-1}-t_{0}<\sigma-t_{0}, S\left(\tau_{n-1}\right)=j\right\}} \mid N(0)=n, S(0)=i, J=4\right] \\
= & \left\{\begin{array}{cl}
\left(G_{n}(s)\right)_{i+1, j} & \text { for } 0 \leq i \leq m-1, \\
\left(G_{n+1}(s) G_{n}(s)\right)_{m j} & \text { for } i=m .
\end{array}\right.
\end{aligned}
$$

Substituting (8)-(11) into (7) yields, for $0 \leq i \leq m$

(12) $\left.G_{n}(s)\right)_{i j}$

$$
=\left((C+n D+s I)_{i i}\right)^{-1}\left((n-1) A_{i j}+\left(B G_{n}(s)\right)_{i j}+\left(E G_{n+1}(s) G_{n}(s)\right)_{i j}\right),
$$


which implies (2).

In a similar way to the derivation of (12), we obtain

$\left(h_{n}(s)\right)_{i}$

$=\left((C+n D+s I)_{i i}\right)^{-1}\left((A \mathbf{1})_{i}+\left(B \boldsymbol{h}_{n}(s)\right)_{i}+\left(E G_{n+1}(s) \boldsymbol{h}_{n}(s)\right)_{i}+\left(E \boldsymbol{h}_{n+1}(s)\right)_{i}\right)$,

which yields (3).

We denote by $g(s)$ the LST of the busy period distribution in the standard $\mathrm{M} / \mathrm{M} / 1$ queue with arrival rate $\lambda$ and service rate $m \mu$. It is well known that for $\operatorname{Re}(s) \geq 0, g(s)$ has a explicit expression

$$
g(s)=\frac{\lambda+m \mu+s-\sqrt{(\lambda+m \mu+s)^{2}-4 \lambda m \mu}}{2 \lambda} .
$$

We give the following theorem without proof, which is very similar to the proof of Theorem 2 in Kim and Kim [15].

Theorem 2. For complex numbers $s$ with $\operatorname{Re}(s) \geq 0$,

$$
\lim _{n \rightarrow \infty} G_{n}(s)=G(s), \quad \lim _{n \rightarrow \infty} \boldsymbol{h}_{n}(s)=\mathbf{0},
$$

where $G(s)$ is the $(m+1) \times(m+1)$ matrix whose $(i, j)$-components, $0 \leq i, j \leq m$, are given by

$$
(G(s))_{i j}=\left\{\begin{array}{cl}
1 & \text { if } 0 \leq j=i+1 \leq m-1, \\
g(s) & \text { if } i=j=m, \\
0 & \text { otherwise. }
\end{array}\right.
$$

\section{Waiting time distribution}

In this section, we study the LSTs of the conditional waiting time and the unconditional waiting time. Let $\left(w_{n}(s)\right)_{i}$ be the conditional LST of $\sigma$, given $N(0)=n \geq 1$ and $S(0)=i, 0 \leq i \leq m$, i.e.,

$$
\left(w_{n}(s)\right)_{i}=\mathbb{E}\left[e^{-s \sigma} \mid N(0)=n, S(0)=i\right], 0 \leq i \leq m
$$

for complex numbers $s$ with $\operatorname{Re}(s) \geq 0$. Let $\boldsymbol{w}_{n}(s)$ be the $(m+1)$-dimensional column vector whose $i$ th component is $\left(w_{n}(s)\right)_{i}$. Then $\boldsymbol{w}_{n}(s)$ satisfies the following theorem.

Theorem 3. For complex numbers $s$ with $\operatorname{Re}(s) \geq 0$,

$$
\begin{aligned}
\boldsymbol{w}_{1}(s) & =\boldsymbol{h}_{1}(s), \\
\boldsymbol{w}_{n}(s) & =\boldsymbol{h}_{n}(s)+G_{n}(s) \boldsymbol{w}_{n-1}(s), n=2,3, \ldots
\end{aligned}
$$

Proof. Since $\sigma \leq \tau_{0}$, we have (13). To derive (14), we write, for $n=2,3, \ldots$,

$$
\begin{aligned}
& \left(w_{n}(s)\right)_{i} \\
= & \mathbb{E}\left[e^{-s \sigma} \mathbb{1}_{\left\{\tau_{n-1} \geq \sigma\right\}} \mid N(0)=n, S(0)=i\right] \\
& +\sum_{j=0}^{m} \mathbb{E}\left[e^{-s \tau_{n-1}} \mathbb{1}_{\left\{\tau_{n-1}<\sigma, S\left(\tau_{n-1}\right)=j\right\}} e^{-s\left(\sigma-\tau_{n-1}\right)} \mid N(0)=n, S(0)=i\right] .
\end{aligned}
$$


The first conditional expectation on the right-hand side of $(15)$ is $\left(h_{n}(s)\right)_{i}$. By the strong Markov property of the Markov process $\left\{\left(N(t), S(t), \mathbb{1}_{\{\sigma \geq t\}}\right): t \geq\right.$ $0\}$, the second conditional expectation on the right-hand side of (15) becomes

$$
\begin{aligned}
& \mathbb{E}\left[e^{-s \tau_{n-1}} \mathbb{1}_{\left\{\tau_{n-1}<\sigma, S\left(\tau_{n-1}\right)=j\right\}} e^{-s\left(\sigma-\tau_{n-1}\right)} \mid N(0)=n, S(0)=i\right] \\
= & \mathbb{E}\left[e^{-s \tau_{n-1}} \mathbb{1}_{\left\{\tau_{n-1}<\sigma, S\left(\tau_{n-1}\right)=j\right\}} \mid N(0)=n, S(0)=i\right] \\
& \times \mathbb{E}\left[e^{-s \sigma} \mid N(0)=n-1, S(0)=j\right] \\
= & \left(G_{n}(s)\right)_{i j}\left(w_{n-1}(s)\right)_{j} .
\end{aligned}
$$

Therefore (15) becomes

$$
\left(w_{n}(s)\right)_{i}=\left(h_{n}(s)\right)_{i}+\sum_{j=0}^{m}\left(G_{n}(s)\right)_{i j}\left(w_{n-1}(s)\right)_{j},
$$

which is the componentwise expression of (14).

The following corollary is immediate from Theorem 3.

Corollary 1. For complex numbers $s$ with $\operatorname{Re}(s) \geq 0$, and $n=1,2, \ldots$,

$$
\boldsymbol{w}_{n}(s)=\sum_{k=1}^{n} G_{n}(s) G_{n-1}(s) \cdots G_{k+1}(s) \boldsymbol{h}_{k}(s) .
$$

Let $w(s)=\mathbb{E}\left[e^{-s \mathcal{W}}\right]$ be the LST of the waiting time of the tagged customer. Then, according to (1), we have the following theorem.

Theorem 4. The LST of the waiting time of an arbitrary customer is given by

$$
w(s)=1-\sum_{n=0}^{\infty} \pi_{n m}+\sum_{n=0}^{\infty} \pi_{n m}\left(w_{n+1}(s)\right)_{m},
$$

for complex numbers $s$ with $\operatorname{Re}(s) \geq 0$.

\section{Numerical computation of the waiting time distribution}

The waiting time distribution can be numerically calculated by inverting the LST of the waiting time distribution. The computational procedure for the waiting time distribution requires three steps:

(1) Calculation of $\pi_{n i}, n \geq 0,0 \leq i \leq m$, the stationary probabilities of the queue size (the number of customers in the orbit and the number of busy servers).

(2) Calculation of the LST of the waiting time distribution.

(3) Inversion of the LST.

The three steps are described in detail below. 


\section{Calculation of the queue size probabilities $\pi_{n i}, n \geq 0,0 \leq i \leq m$}

For the calculation of the queue size distribution, we use the method proposed by Neuts and Rao [18]. For a positive integer $k$ that will be determined later, let

$$
Q^{(k)}=\left[\begin{array}{cccccccc}
B-C & E & & & & & & \\
\\
A & B-C-D & E & & & & & \\
& 2 A & B-C-2 D & E & & & & \\
& & \ddots & \ddots & \ddots & & & \\
& & & k A & B-C-k D & E & \\
& & & & k A & B-C-k D & E & \\
& & & & & \ddots & \ddots & \ddots
\end{array}\right] .
$$

Let $\pi_{n i}^{(k)}, n \geq 0,0 \leq i \leq m$, be the stationary probabilities of a continuous time Markov process with the infinitesimal generator $Q^{(k)}$ and let $\boldsymbol{\pi}_{n}^{(k)}=$ $\left(\pi_{n 0}^{(k)}, \ldots, \pi_{n m}^{(k)}\right), n \geq 0$. According to the homogeneity of the transition rates from levels above $k$, we have

$$
\boldsymbol{\pi}_{n}^{(k)}=\boldsymbol{\pi}_{k}^{(k)}\left(R^{(k)}\right)^{n-k} \text { for } n \geq k,
$$

where $R^{(k)}$ is the minimal nonnegative solution of the quadratic matrix equation

$$
E+R^{(k)}(B-C-k D)+k\left(R^{(k)}\right)^{2} A=O .
$$

The probabilities $\pi_{n i}^{(k)}, 0 \leq n \leq k, 0 \leq i \leq m$, can be easily computed by the matrix analytic method, see Neuts and Rao [18] for the detailed computation. Therefore, $\pi_{n i}^{(k)}$ is used as an approximation of $\pi_{n i}, n \geq 0,0 \leq i \leq m$, where $k$ is chosen so that

$$
\sum_{n=k+1}^{\infty} \sum_{i=0}^{m} \pi_{n i}^{(k)}=\boldsymbol{\pi}_{k}^{(k)} R^{(k)}\left(I-R^{(k)}\right)^{-1} \mathbf{1}<\epsilon
$$

for a predetermined small $\epsilon>0$.

\section{Calculation of the LST of the waiting time distribution}

By Theorem $1, G_{n}(s)$ and $\boldsymbol{h}_{n}(s)$ can be calculated if we know $G_{n+1}(s)$ and $\boldsymbol{h}_{n+1}(s)$. Moreover, by Theorem $2, G_{n}(s)$ and $\boldsymbol{h}_{n}(s)$ can be approximated by $G(s)$ and $\mathbf{0}$, respectively, for large $n$. Based on the observations, we calculate approximations $G_{n}^{(k)}(s)$ and $\boldsymbol{h}_{n}^{(k)}(s)$ for $G_{n}(s)$ and $\boldsymbol{h}_{n}(s), n=0,1,2, \ldots$, by the recursion:

(i) For $n \geq 2 k+1$,

$$
\begin{aligned}
& G_{n}^{(k)}(s)=G(s), \\
& \boldsymbol{h}_{n}^{(k)}(s)=\mathbf{0} .
\end{aligned}
$$


(ii) For $n=2 k, 2 k-1, \ldots, 0$,

$$
\begin{aligned}
& G_{n}^{(k)}(s)=(n-1)\left[C+n D-B-E G_{n+1}^{(k)}(s)+s I\right]^{-1} A, \\
& \boldsymbol{h}_{n}^{(k)}(s)=\left[C+n D-B-E G_{n+1}^{(k)}(s)+s I\right]^{-1}\left(A \mathbf{1}+E \boldsymbol{h}_{n+1}^{(k)}(s)\right) .
\end{aligned}
$$

According to Theorem 3 , we obtain approximation $\boldsymbol{w}_{n}^{(k)}(s)$ for $\boldsymbol{w}_{n}(s), n=$ $1,2, \ldots$, by the following recursion:

$$
\begin{aligned}
\boldsymbol{w}_{1}^{(k)}(s) & =\boldsymbol{h}_{1}^{(k)}(s), \\
\boldsymbol{w}_{n}^{(k)}(s) & =\boldsymbol{h}_{n}^{(k)}(s)+G_{n}^{(k)}(s) \boldsymbol{w}_{n-1}^{(k)}(s), n=2,3, \ldots .
\end{aligned}
$$

Finally, by Theorem 4 and (16), the LST $w(s)$ of the waiting time distribution is approximated by

$$
\begin{aligned}
w^{(k)}(s) & =1-\sum_{n=0}^{k} \pi_{n m}^{(k)}+\sum_{n=0}^{k} \pi_{n m}^{(k)}\left(w_{n+1}^{(k)}(s)\right)_{m} \\
& =1+\sum_{n=0}^{k} \pi_{n m}^{(k)}\left(\left(w_{n+1}^{(k)}(s)\right)_{m}-1\right) .
\end{aligned}
$$

\section{Inversion of the LST}

We use the Euler method for the numerical inversion of the LST of the waiting time distribution. For a detailed description of the Euler method for the numerical inversion of LSTs, see Abate and Whitt [1].

TABLE 1 . Waiting time distribution $\mathbb{P}(\mathcal{W}>x)$ when $\nu=0.5$, $\rho=0.7$.

\begin{tabular}{|c|cc|cc|}
\hline$m$ & \multicolumn{2}{|c|}{2} & \multicolumn{2}{c|}{5} \\
\hline$x$ & Simul. & Ours & Simul. & Ours \\
\hline 0 & 0.53604 & 0.53604 & 0.31120 & 0.31120 \\
1 & 0.47353 & 0.47349 & 0.27088 & 0.27090 \\
3 & 0.33821 & 0.33824 & 0.17950 & 0.17953 \\
5 & 0.24372 & 0.24380 & 0.11888 & 0.11888 \\
10 & 0.11336 & 0.11341 & 0.04560 & 0.04562 \\
15 & 0.05603 & 0.05610 & 0.01930 & 0.01932 \\
20 & 0.02904 & 0.02909 & 0.00881 & 0.00883 \\
25 & 0.01562 & 0.01566 & 0.00426 & 0.00428 \\
30 & 0.00866 & 0.00869 & 0.00215 & 0.00218 \\
35 & 0.00491 & 0.00494 & 0.00113 & 0.00115 \\
40 & 0.00285 & 0.00288 & 0.00061 & 0.00063 \\
45 & 0.00168 & 0.00171 & 0.00034 & 0.00036 \\
50 & 0.00102 & 0.00104 & 0.00019 & 0.00021 \\
\hline
\end{tabular}

\begin{tabular}{|c|cc|cc|}
\hline$m$ & \multicolumn{2}{|c|}{10} & \multicolumn{2}{c|}{50} \\
\hline$x$ & Simul. & Ours & Simul. & Ours \\
\hline 0 & 0.16828 & 0.16828 & 0.00749 & 0.00749 \\
1 & 0.14576 & 0.14580 & 0.00647 & 0.00647 \\
3 & 0.09399 & 0.09402 & 0.00408 & 0.00409 \\
5 & 0.06009 & 0.06012 & 0.00253 & 0.00254 \\
7 & 0.03900 & 0.03904 & 0.00160 & 0.00160 \\
9 & 0.02582 & 0.02585 & 0.00102 & 0.00103 \\
11 & 0.01744 & 0.01746 & 0.00067 & 0.00068 \\
13 & 0.01199 & 0.01201 & 0.00045 & 0.00046 \\
15 & 0.00838 & 0.00840 & 0.00031 & 0.00031 \\
17 & 0.00594 & 0.00596 & 0.00021 & 0.00022 \\
20 & 0.00363 & 0.00365 & 0.00013 & 0.00013 \\
25 & 0.00169 & 0.00171 & 0.00006 & 0.00006 \\
30 & 0.00083 & 0.00084 & 0.00003 & 0.00003 \\
\hline
\end{tabular}


TABLE 2. Waiting time distribution $\mathbb{P}(\mathcal{W}>x)$ when $\nu=0.5$, $\rho=0.9$.

\begin{tabular}{|c|cc|cc|}
\hline$m$ & \multicolumn{2}{|c|}{2} & \multicolumn{2}{c|}{5} \\
\hline$x$ & Simul. & Ours & Simul. & Ours \\
\hline 0 & 0.82507 & 0.82507 & 0.69178 & 0.69178 \\
1 & 0.77983 & 0.77987 & 0.64434 & 0.64441 \\
3 & 0.68308 & 0.68328 & 0.53837 & 0.53848 \\
5 & 0.60227 & 0.60249 & 0.45426 & 0.45440 \\
7 & 0.53422 & 0.53441 & 0.38769 & 0.38774 \\
10 & 0.45049 & 0.45065 & 0.31097 & 0.31099 \\
15 & 0.34582 & 0.34602 & 0.22303 & 0.22310 \\
20 & 0.27070 & 0.27090 & 0.16541 & 0.16534 \\
30 & 0.17314 & 0.17327 & 0.09708 & 0.09705 \\
40 & 0.11541 & 0.11553 & 0.06048 & 0.06054 \\
50 & 0.07935 & 0.07942 & 0.03932 & 0.03941 \\
60 & 0.05587 & 0.05591 & 0.02644 & 0.02648 \\
70 & 0.04002 & 0.04012 & 0.01820 & 0.01826 \\
\hline
\end{tabular}

\begin{tabular}{|c|cc|cc|}
\hline$m$ & \multicolumn{2}{|c|}{10} & \multicolumn{2}{c|}{50} \\
\hline$x$ & Simul. & Ours & Simul. & Ours \\
\hline 0 & 0.57765 & 0.57765 & 0.29031 & 0.29031 \\
1 & 0.53496 & 0.53504 & 0.26759 & 0.26762 \\
3 & 0.43771 & 0.43789 & 0.21495 & 0.21501 \\
5 & 0.36204 & 0.36218 & 0.17451 & 0.17460 \\
7 & 0.30351 & 0.30365 & 0.14392 & 0.14402 \\
10 & 0.23809 & 0.23820 & 0.11064 & 0.11072 \\
15 & 0.16589 & 0.16604 & 0.07516 & 0.07522 \\
20 & 0.12018 & 0.12036 & 0.05341 & 0.05349 \\
30 & 0.06826 & 0.06833 & 0.02952 & 0.02952 \\
40 & 0.04154 & 0.04158 & 0.01758 & 0.01760 \\
50 & 0.02653 & 0.02653 & 0.01104 & 0.01105 \\
60 & 0.01754 & 0.01753 & 0.00719 & 0.00720 \\
70 & 0.01192 & 0.01191 & 0.00483 & 0.00484 \\
\hline
\end{tabular}

TABLE 3. Waiting time distribution $\mathbb{P}(\mathcal{W}>x)$ when $\nu=2$, $\rho=0.7$.

\begin{tabular}{|c|cc|cc|}
\hline$m$ & \multicolumn{2}{|c|}{2} & \multicolumn{2}{c|}{5} \\
\hline$x$ & Simul. & Ours & Simul. & Ours \\
\hline 0 & 0.55308 & 0.55308 & 0.34441 & 0.34441 \\
1 & 0.40251 & 0.40254 & 0.24532 & 0.24539 \\
3 & 0.20578 & 0.20578 & 0.11797 & 0.11802 \\
5 & 0.11776 & 0.11780 & 0.06483 & 0.06484 \\
7 & 0.07218 & 0.07220 & 0.03855 & 0.03859 \\
9 & 0.04631 & 0.04632 & 0.02415 & 0.02419 \\
11 & 0.03069 & 0.03073 & 0.01569 & 0.01575 \\
13 & 0.02088 & 0.02092 & 0.01051 & 0.01055 \\
15 & 0.01452 & 0.01454 & 0.00720 & 0.00723 \\
17 & 0.01025 & 0.01029 & 0.00502 & 0.00505 \\
20 & 0.00625 & 0.00629 & 0.00301 & 0.00304 \\
25 & 0.00291 & 0.00293 & 0.00136 & 0.00139 \\
30 & 0.00143 & 0.00145 & 0.00066 & 0.00068 \\
\hline
\end{tabular}

\begin{tabular}{|c|cc|cc|}
\hline$m$ & \multicolumn{2}{|c|}{10} & \multicolumn{2}{c|}{50} \\
\hline$x$ & Simul. & Ours & Simul. & Ours \\
\hline 0 & 0.19618 & 0.19618 & 0.00939 & 0.00939 \\
1 & 0.13882 & 0.13880 & 0.00660 & 0.00661 \\
3 & 0.06539 & 0.06534 & 0.00305 & 0.00306 \\
5 & 0.03541 & 0.03539 & 0.00163 & 0.00164 \\
7 & 0.02088 & 0.02085 & 0.00096 & 0.00096 \\
9 & 0.01298 & 0.01297 & 0.00059 & 0.00060 \\
11 & 0.00838 & 0.00839 & 0.00038 & 0.00038 \\
13 & 0.00558 & 0.00559 & 0.00025 & 0.00026 \\
15 & 0.00381 & 0.00382 & 0.00017 & 0.00018 \\
17 & 0.00265 & 0.00266 & 0.00012 & 0.00012 \\
20 & 0.00157 & 0.00159 & 0.00007 & 0.00008 \\
25 & 0.00071 & 0.00072 & 0.00003 & 0.00004 \\
30 & 0.00034 & 0.00035 & 0.00001 & 0.00002 \\
\hline
\end{tabular}

\section{Numerical results}

In this section, numerical examples are presented. We consider the $\mathrm{M} / \mathrm{M} / \mathrm{m}$ retrial queue with $m=2,5,10,50$ and obtain the waiting time distribution numerically using the method described in the previous section. The exogenous arrival rate is set to $\lambda=1$. We take $\epsilon=10^{-10}$ for approximations of the LSTs of the waiting time distribution. In Tables 1-4, we present the simulation results and numerical results by our method for the tail probabilities of the waiting time $\mathcal{W}$, when $\nu=0.5, \nu=2$ and $\rho=0.7, \rho=0.9$. In the tables, we 
TABLE 4. Waiting time distribution $\mathbb{P}(\mathcal{W}>x)$ when $\nu=2$, $\rho=0.9$.

\begin{tabular}{|c|cc|cc|}
\hline$m$ & \multicolumn{2}{|c|}{2} & \multicolumn{2}{c|}{5} \\
\hline$x$ & Simul. & Ours & Simul. & Ours \\
\hline 0 & 0.55308 & 0.55308 & 0.34441 & 0.34441 \\
1 & 0.40251 & 0.40254 & 0.24532 & 0.24539 \\
3 & 0.20578 & 0.20578 & 0.11797 & 0.11802 \\
5 & 0.11776 & 0.11780 & 0.06483 & 0.06484 \\
7 & 0.07218 & 0.07220 & 0.03855 & 0.03859 \\
9 & 0.04631 & 0.04632 & 0.02415 & 0.02419 \\
11 & 0.03069 & 0.03073 & 0.01569 & 0.01575 \\
13 & 0.02088 & 0.02092 & 0.01051 & 0.01055 \\
15 & 0.01452 & 0.01454 & 0.00720 & 0.00723 \\
17 & 0.01025 & 0.01029 & 0.00502 & 0.00505 \\
20 & 0.00625 & 0.00629 & 0.00301 & 0.00304 \\
25 & 0.00291 & 0.00293 & 0.00136 & 0.00139 \\
30 & 0.00143 & 0.00145 & 0.00066 & 0.00068 \\
\hline
\end{tabular}

\begin{tabular}{|c|cc|cc|}
\hline$m$ & \multicolumn{2}{|c|}{10} & \multicolumn{2}{c|}{50} \\
\hline$x$ & Simul. & Ours & Simul. & Ours \\
\hline 0 & 0.19618 & 0.19618 & 0.00939 & 0.00939 \\
1 & 0.13882 & 0.13880 & 0.00660 & 0.00661 \\
2 & 0.09330 & 0.09324 & 0.00439 & 0.00440 \\
3 & 0.06539 & 0.06534 & 0.00305 & 0.00306 \\
4 & 0.04748 & 0.04745 & 0.00220 & 0.00221 \\
5 & 0.03541 & 0.03539 & 0.00163 & 0.00164 \\
7 & 0.02088 & 0.02085 & 0.00096 & 0.00096 \\
9 & 0.01298 & 0.01297 & 0.00059 & 0.00060 \\
11 & 0.00838 & 0.00839 & 0.00038 & 0.00038 \\
13 & 0.00558 & 0.00559 & 0.00025 & 0.00026 \\
15 & 0.00381 & 0.00382 & 0.00017 & 0.00018 \\
17 & 0.00265 & 0.00266 & 0.00012 & 0.00012 \\
20 & 0.00157 & 0.00159 & 0.00007 & 0.00008 \\
\hline
\end{tabular}

use the Euler method developed by Abate and Whitt [1] to invert the LSTs numerically. The simulation results are obtained by generating $10^{7}$ positive waiting times. Tables 1-4 demonstrate that the numerical results obtained by our method are consistent with those obtained by simulation.

\section{References}

[1] I. Abate and W. Whitt, The Fourier-series method for inverting transforms of probability distributions, Queueing Systems Theory Appl. 10 (1992), no. 1-2, 5-87.

[2] J. R. Artalejo, A classified bibliography of research on retrial queues: Progress in 19901999, Top 7 (1999), no. 2, 187-211.

[3] _ Accessible bibliography on retrial queues, Math. Comput. Model. 30 (1999), 1-6.

[4] Accessible bibliography on retrial queues: progress in 2000-2009, Math. Comput. Modelling 51 (2010), no. 9-10, 1071-1081.

[5] J. R. Artalejo and A. Gómez-Corral, Waiting time in the $M / M / c$ queue with finite retrial group, Bull. Kerala Math. Assoc. 2 (2005), no. 1, 1-17.

[6] Retrial Queueing Systems, Springer, 2008.

[7] Q. H. Choo and B. Conolly, New results in the theory of repeated orders queueing systems, J. Appl. Probab. 16 (1979), no. 3, 631-640.

[8] G. I. Falin, On the waiting time in a single-channel queueing system with secondary calls, Moscow Univ. Comput. Math. Cybernet. 4 (1977), 83-87.

[9] _ A survey of retrial queues, Queueing Systems Theory Appl. 7 (1990), no. 2, $127-167$.

[10] G. I. Falin and J. G. C. Templeton, Retrial Queues, Chapman \& Hall, London, 1997.

[11] B. S. Greenberg and R. W. Wolff, An upper bound on the performance of queues with returning customers, J. Appl. Probab. 24 (1987), no. 2, 466-475.

[12] T. Hanschke, A computational procedure for the variance of the waiting time in the $M / M / 1 / 1$ queue with repeated attempts, In: Operations Research Proceedings, 525532, Springer-Verlag, Berlin, 1986.

[13] - Explicit formulas for the characteristics of the $M / M / 2 / 2$ queue with repeated attempts, J. Appl. Probab. 24 (1987), no. 2, 486-494. 
[14] J. Keilson, J. Cozzolino, and H. Young, A service system with unfilled requests repeated, Oper. Res. 16 (1968), 1126-1137.

[15] J. Kim and B. Kim, Waiting time distribution in an $M / P H / 1$ retrial queue, Performance Evaluation $\mathbf{7 0}$ (2013), 286-299.

[16] V. G. Kulkarni, Letters to the editor, J. Appl. Probab. 19 (1982), 901-905.

[17] V. G. Kulkarni and H. M. Liang, Retrial queues revisited, In: Frontiers in Queueing: Models and Applications in Science and Engineering (J. H. Dshalalow, ed.), 19-34. CRC Press, Boca Raton, 1997.

[18] M. F. Neuts and B. M. Rao, Numerical investigation of a multiserver retrial model, Queueing Systems Theory Appl. 7 (1990), 169-190.

[19] R. I. Wilkinson, Theories for toll traffic engineering in the U.S.A., The Bell System Technical Journal 35 (1956), 421-514.

[20] T. Yang and J. G. C. Templeton, A survey on retrial queues, Queueing Systems Theory Appl. 2 (1987), no. 3, 201-233.

JEONGSIM KIM

Department of Mathematics Education

Chungbuk National University

Cheonguu 361-763, Korea

E-mail address: jeongsimkim@chungbuk.ac.kr

JERIM KIM

Department of Business Administration

YongIN UNIVERSITY

YONGIN 449-714, KoREA

E-mail address: jerimkim@yongin.ac.kr 